\title{
Cultural differences between the countries of Western Europe and the countries of the ancient Eastern Bloc
}

\author{
[Les différences culturelles entre les pays d'Europe occidentale et les \\ pays de l'ancien bloc de l'Est]
}

\author{
Ludmila Meskova
}

DOI: 10.18355/XL.2018.11.01XL.25

\begin{abstract}
The aim of this article is to show the cultural differences that exist between Western European countries and those of the former Eastern Bloc. On the one hand, we will study the consequences of the different political regimes and on the other hand those connected to the heritage of different cultures. We will touch on the example of the French (descendants of the Latins) and the Slovaks as representatives of the Slavic countries. On a professional level, we will emphasize the observed differences concerning the methods of behaviour, of thinking, of reasoning, of argumentation and of discussion as well as such notions as motivation, time, assertiveness and even emotional dimensions. These observations and personal experiences are based on formal surveys, inquiries and interviews with French and Slovak employees who work in French businesses located in Slovakia or by the work of French and Slovak students studying in Slovakia or in France. The results of this research can be applied in education as well as in organisations or businesses whose workforce is composed of several nationalities or cultures.
\end{abstract}

Key words: intercultural communication, cultural difference, cultural conflict, cultural sensitivity, stereotypes

\section{Résumé}

Le but de notre article est de démontrer les différences culturelles existant entre les pays d'Europe occidentale et ceux de l'ancien bloc de l'Est et ce malgré l'existence d'un berceau commun. Nous étudierons, d'un côté, les incidences des différents régimes politiques sur ces pays et, de l'autre, prendrons en considération leurs héritages culturels différents. Nous nous appuierons pour cela sur l'exemple des Français, que nous considérerons comme une nation latine, et sur celui des Slovaques, peuple slave. Au niveau professionnel, nous mettrons en relief les différences observées concernant la façon de réfléchir, de raisonner, d'agir, de faire son planning, d'argumenter, ainsi que des notions telles que la motivation, le temps, l'assertivité ou encore la dimension émotionnelle. Ces observations issues d'expériences personnelles sont appuyées par des sondages, enquêtes et entretiens avec des Français et des Slovaques travaillant dans des entreprises françaises implantées en Slovaquie ou par les travaux d'étudiants français et slovaques faisant leurs études en Slovaquie ou en France. Les résultats de notre étude pourront être utilisés dans l'enseignement et appliqués également à des organismes ou entreprises dont l'effectif comporte des nationalités et cultures différentes.

Mots-clés: communication interculturelle, différence culturelle, conflit culturel, sensibilité culturelle, stéréotypes

\section{Introduction}

Nous vivons dans un environnement de plus en plus complexe qui se mondialise. Les sociétés d'aujourd'hui se transforment peu à peu en organisations multiculturelles. Au travers de ces multinationales, nous découvrons ainsi d'autres cultures, d'autres identités, d'autres religions. Les échanges internationaux sont 
marqués par la problématique de l'interculturel. Selon Goulvestre (2012: 12), l'interculturel est une notion d'échanges, de partages, d'attention envers l'autre, de croisements et de sensibilités. Stoiciu (2011: 60, 67, 68) tente de définir la communication interculturelle à partir du préfixe inter (du mot interculturel) qui peut être chargé de trois enjeux majeurs de la communication : il s'agit d'une rencontre avec, d'une rencontre entre et d'une rencontre agissant sur. La communication interculturelle est liée aux modalités de communication (verbale ou non verbale), aux filtres perceptifs (stéréotypes, préjugés et ethnocentrisme), aux barrages sociaux (formes de discrimination), ainsi qu'aux zones sensibles à la différence (perception $\mathrm{du}$ temps, de l'espace, des codes de sociabilité, rituels de passage). Ce sont les zones sensibles à la différence, c'est-à-dire les différences culturelles, qui attirent ici notre attention.

Nous partageons l'avis de Hermesse (2005) qui affirme qu'il n'est pas possible de fournir un mode d'emploi universel de comportement dans la conduite des affaires internationales.

En dépit de leurs racines européennes communes, des différences culturelles entre les pays d'Europe occidentale et ceux de l'ancien bloc de l'Est existent toujours. Sous la notion de pays occidentaux, nous comprenons l'ensemble des États capitalistes d'Europe de l'Ouest avant la chute du Mur de Berlin par opposition aux pays de l'Est, d'économie socialiste. Après 1989, la transition de l'économie planifiée vers l'économie de marché ne s'est pas faite du jour au lendemain. Cette transition est un processus de longue durée qui ne s'est pas encore achevé. Selon Horehájová Marasová (2006: 349), le passage à l'économie de marché, après 40 ans de planification et de gestion centralisées représentait, et continue à représenter, un processus complexe. Il s'agissait de la transformation de la propriété étatique en propriété privée. Notre étude ne s'attachera pas à décrire tous les aspects de ce processus. Notre attention se portera en particulier sur la «transition » au niveau de l'attitude et de la pensée des citoyens, tant dans les pays d'Europe de l'Ouest que dans les pays d'Europe de l'Est. Les deux blocs ont fait face à des obstacles qu'ils ont dû surmonter. Il a alors fallu se battre avec les préjugés et les fausses idées que chacun se faisait de l'autre.

Nous nous appuierons sur l'exemple des Français en tant que représentants d'un pays d'Europe de l'Ouest et des Slovaques en tant que représentants d'un pays de l'ancien bloc de l'Est. Les systèmes économiques et politiques des deux pays étaient, en effet, entièrement différents avant 1989. Il est vrai que la mondialisation joue à présent un rôle important, car elle tend à standardiser l'être humain, à prédire son comportement mais aussi, d'une certaine manière, à le manipuler. Les grands pays désirent conserver la différence qu'ils détiennent avec les pays moins développés afin de maintenir une sorte de sentiment de supériorité. La supériorité nationale ressentie par un individu peut constituer un obstacle à la communication interculturelle en ce sens qu'elle empêche toute critique de son propre groupe culturel et ferme l'individu à la découverte et à l'acceptation d'une culture différente, pourtant fondamentale si l'on souhaite ouvrir son esprit et améliorer sa propre perspective culturelle. Ceci est surtout valable dans les relations personnelles, moins dans les relations professionnelles, car si des entreprises multinationales ou des organismes à caractère multinational veulent fonctionner correctement, ils doivent se respecter l'un l'autre et ce autant que possible.

Nos observations sont issues des expériences personnelles ayant jalonné notre carrière professionnelle en tant qu'universitaire se consacrant à la problématique de l'interculturel. Ces observations ont été appuyées par des sondages, enquêtes et entretiens avec des Français et des Slovaques travaillant dans des entreprises françaises implantées en Slovaquie ou par les travaux d'étudiants français et slovaques faisant leurs études en Slovaquie ou en France. 
Nous nous concentrerons sur deux aspects. Nous étudierons, d'un côté, les incidences des différents régimes politiques sur ces pays et, de l'autre, les différences culturelles plus anciennes marquant ces deux pays, comme, par exemple, l'héritage latin des Français et l'origine slave des Slovaques.

\section{Les différences liées aux systèmes politiques}

Nous essaierons tout d'abord de répondre aux questions suivantes : ces différences sont-elles dues à une différence d'évolution des nations en question et à leurs différents systèmes politiques?

La communication internationale et interculturelle est historiquement et théoriquement liée au développement. Ce concept a été utilisé en politique et en sciences sociales depuis les années 1950 pour faire référence aux progrès économiques dus à la technologie. Cette tendance imposait la culture occidentale comme modèle unique à suivre (Rico de Sotelo - Serpereau, 2011: 97-98). L'opposition entre bloc occidental et bloc de l'Est (bloc soviétique) a eu un impact sur les orientations politiques prises par les deux pays, avec une Europe de l'Ouest, y compris la France, quasiment alignée sur l'impérialisme américain, alors que le reste de l'Europe, et donc la Slovaquie (ex-Tchécoslovaquie) était sous l'influence politique et économique de l'URSS, adoptant ainsi le modèle communiste (dès 1948). Deux approches politiques et économiques littéralement opposées, une Europe coupée en deux par le rideau de fer et un développement à deux vitesses.

La première différence est évidente : les Français et les Slovaques ont vécu dans des pays dont les régimes politiques étaient en contradiction. La France est un État démocratique qui n'a pas connu la dictature communiste, la Slovaquie (un des deux États de l'ex-Tchécoslovaquie) est devenue un État indépendant en 1993, après avoir connu la dictature communiste (de 1948 à 1989). Les deux systèmes politiques étaient en opposition, tout comme leurs systèmes économiques ainsi que leurs styles de vie. L'économie de marché en France et l'économie planifiée en Slovaquie jusqu'en 1989 ont marqué profondément le comportement et les mentalités de leurs habitants. Les effets de la société de consommation ne sont pas les mêmes dans les deux pays. En ce qui concerne la Slovaquie, bien que différents de leurs aînés, les jeunes Slovaques sont pourtant également différents des jeunes Français. Pourquoi ? Ils ont été élevés, éduqués et formés par la génération qui a connu le régime précédent. Ce système politique différent a laissé des traces sur leurs parents, sur les enseignants qui transmettent involontairement les habitudes acquises à la génération suivante. La nouvelle génération est la génération du changement, de la transition.

Après la chute du Mur de Berlin, l'influence du régime précédent sur les Slovaques et la propagande politique en France jusqu' en 1989 se sont reflétées dans le comportement des deux groupes.

Les jeunes Slovaques s'intéressent très peu et sont peu concernés par la politique à la différence des jeunes Français. Pourquoi ? L'orientation politique des générations précédentes (parents et grands-parents de la jeune génération) a été imposée pendant 40 ans jusqu'en 1989. Il n'était alors pas recommandé de trop réfléchir ou de s'opposer à l'idéologie prescrite par le régime communiste. Le désintérêt des jeunes d'aujourd'hui vis-à-vis de la vie politique trouvent ses racines dans un passé récent.

Il existe une différence primordiale entre les Français et les Slovaques. Il est, en effet, difficile de s'imaginer la peur des citoyens de l'ancien bloc de l'Est traversant la frontière "occidentale » sous le régime précédent et notamment la peur d'être contrôlé parce qu'on disposait d'une somme plus importante que celle prescrite et autorisée par le régime. Il était très humiliant d'aller en Occident avec la somme accordée par l'Etat qui s'avérait insuffisante. À la frontière, la police contrôlait tout : la presse, les livres, la quantité de monnaie étrangère, la raison du déplacement... L'État permettait à ses ressortissants de partir en voyage privé ou en déplacement 
professionnel pour une courte période, mais ceux-ci devaient laisser leurs enfants ou leur conjoint dans leur pays d'origine. L'État (ou la police) s'assurait ainsi de leur retour. Presque 30 ans après, cette peur lors du passage de la frontière persiste à ceux qui l'ont vécue. Le même sentiment surgit malgré eux. Étrange ? Non, ils restent tout simplement marqués, les traces du régime politique précédent persistent. Cet exemple montre bien de quelle façon l'ancienne génération qui a connu le régime précédent a pu influencer, contre son gré, les générations suivantes et pourquoi les jeunes Slovaques ou représentants d'autres pays de l'ancien bloc de l'Est ne peuvent pas être tout à fait les mêmes que les jeunes Français ou d'autres jeunes d'Europe occidentale.

En Slovaquie, beaucoup de personnes orientent leurs dépenses de consommation vers des biens de consommation divers (automobile, smartphones,...). Tous les étrangers constatent que les Slovaques aiment posséder une belle voiture, ce qui leur paraît surprenant. Ils s'endettent pour avoir une voiture de luxe. Quelle explication peut-on donner à cela ? Cette possibilité n'existait pas pour les Slovaques avant 1989. Le régime précédent était caractérisé par un certain nivelage. Tout était mis au même niveau, gris, peu varié, les modèles disponibles étaient sans fantaisie et le nombre de marques limité comme par exemple la Skoda (Tchécoslovaquie), la Ziguli (URSS), la Trabant (Allemagne de l'Est).

Certains pays européens étaient et continuent à être complètement méconnus par la plupart des Français. C'est notamment le cas de la Slovaquie. Dans les années 60, on ne savait même pas quelle était la capitale de la Tchécoslovaquie. Belgrade ? Budapest ? Prague ? Aujourd'hui encore, beaucoup de Français confondent la Slovaquie et la Slovénie, ils pensent que la séparation des Tchèques et des Slovaques est intervenue après une guerre en Tchécoslovaquie en 1989. Et pourtant l'ex-Tchécoslovaquie est située assez loin des Balkans (ex-Yougoslavie) et donc de la zone de conflit armé au moment du démantèlement des États de ce pays. Rouet- Rošteková (2013: 48) constatent que la Tchécoslovaquie a disparu dans le calme. Les Français qui viennent en Slovaquie pensent venir dans un pays très pauvre où ils ne trouveront pas ce qu'on trouve en Europe. Bien sûr, le niveau de vie reste inférieur à celui que l'on trouve en France. Mais ils sont agréablement surpris en découvrant de grands centres commerciaux, une population accueillante qui aime bien s'habiller et suit la mode européenne ou mondiale, peut étudier dans de bonnes conditions ou travailler aussi dans des firmes internationales. Les touristes apprécient de pouvoir trouver des produits du terroir et du bon vin, dont les cuvées ont remporté des médailles d'or ou d'argent dans les concours en France ces dernières années. Les Français ignorent d'ailleurs que le vin de Tokay était le préféré de Louis XIV. La Slovaquie possède un tiers de ces vignes qu'elle partage avec la Hongrie.

Selon nous, la propagande politique en France pendant la Guerre froide a influencé la pensée des gens d'une façon importante, ce qui explique cette méconnaissance et parfois même une certaine méfiance. Nous rejoignons sur ce point Suchá (2013: 167) qui constate que la Guerre froide a approfondi le conflit entre l'Est et l'Ouest, entre le système basé sur le communisme et le système basé sur la démocratie libérale. Elle a donc laissé des traces. Aujourd'hui, les Français manquent encore d'informations sur ce pays, car peu de promotion est faite pour attirer les touristes, pour découvrir ses montagnes, ses lacs, ses grottes ainsi que sa population avec son histoire, ses habitudes et ses coutumes.

Il faut dire que les Slovaques connaissent beaucoup mieux la France que les Français la Slovaquie. Les petits pays connaissent d'habitude mieux les pays beaucoup plus grands. Selon Rouet - Rošteková (2013: 8), le visiteur étranger, surtout quand il voyage hors de la capitale, découvre vite que les Slovaques sont curieux de l'Europe, de ses cultures, de ses politiques comme de ses traditions et de ses contradictions. 
Le gouvernement slovaque devrait mettre tout en œuvre pour essayer d'attirer des touristes venant d'Europe de l'Ouest. L'histoire slovaque, tout comme celle des autres pays d'Europe centrale et orientale, gagneraient à être découvertes, tout comme les valeurs qu'elles véhiculent, celles-ci pouvant beaucoup apporter sur le plan des relations humaines.

Nous avons présenté ici quelques différences entre la Slovaquie et la France. Chaque pays de l'ancien bloc de l'Est avait ses spécificités, ses propres caractéristiques. Les souffrances des habitants de la Slovaquie (ex-Tchécoslovaquie, mais aussi celles des citoyens de Roumanie, de Bulgarie, d'Albanie, de Pologne, de Hongrie et d'ex-Yougoslavie) n'étaient pas les mêmes. On trouvait certes des différences, mais dans l'ensemble, la dictature communiste a eu des effets néfastes sur la population de ces pays ( « lavage de cerveaux », persécution des chrétiens et des opposants au régime, emprisonnement de ceux qui ne convenaient pas au régime politique ou qui osaient s'exprimer librement).

\section{Les différences liées aux héritages culturels}

L'héritage culturel influence notre vision du temps, de l'espace et des relations entre les hommes (Sickova, 2008: 171). Selon Slatinská - Pecníková (2017: 22), l'identité interculturelle permet de dépasser l'identité culturelle qui est, à la différence de la première, liée à une seule culture. Il n'est pas toujours évident de surmonter ce qui est profondément ancré dans notre propre culture et d'arriver à voir l'autre sous l'optique de sa culture. Des comportements jugés normaux dans une culture peuvent paraître complètement anormaux dans d'autres (Goulvestre, 2012: 21). Pour éviter les malentendus, il faut prendre en compte ces différences.

$\mathrm{Au}$ niveau professionnel, nous mettrons en relief les différences concernant la façon de réfléchir, d'agir, de faire son planning, d'argumenter, etc. Nous nous concentrerons ici sur quelques traits spécifiques qui différencient les représentants de deux nations.

Les Français sont habitués à bien construire leur plan, ils argumentent et justifient tout selon une structure qui leur est propre (Meskova, 2007: 56-65). C'est l'héritage de la «pensée cartésienne » (Kalika (2005: 57). Les Slovaques sont aussi structurés et sont capables d'argumenter, ils le font intuitivement sans avoir appris à le faire systématiquement, à la différence des Français qui y ont été formés tout au long de leur scolarité. Étant donné le régime politique précédent, la formation et le système d'enseignement n'ont pas mis l'accent sur la méthodologie de structuration et d'argumentation en Slovaquie.

Les Français s'appliquent à toujours bien préparer leurs rencontres ou négociations. Ils peuvent parfois donner l'impression de tenir des propos n'ayant aucun lien les uns avec les autres, de sauter d'un sujet à l'autre alors que ce mode de négociation, avec ses changements de sujet, ses interruptions et ses prises de tours de parole chaotiques, constitue en fait autant de façons de juger leurs interlocuteurs : c'est ce qu'on appelle la «négociation à la française ».

Les Slovaques ne raisonnent pas de la même façon que les Français. Ils réfléchissent plus à court et moyen terme tandis que les Français préfèrent avoir une vision d'ensemble et anticiper sur le long terme. Les Slovaques préfèrent analyser que synthétiser. Les Français synthétisent plus facilement après une bonne analyse.

Les manageurs français pensent que les Slovaques sont flexibles, qu'ils s'adaptent vite à de nouvelles conditions et surmontent assez rapidement les difficultés. Ils les considèrent de plus comme étant très travailleurs et assumant bien leurs responsabilités. Ces caractéristiques sont positives. La preuve en est apportée par le nombre de jeunes manageurs slovaques occupant des postes de cadres supérieurs dans des entreprises transnationales tant à l'étranger que dans le pays.

Les cultures fondées sur la collectivité n'accordent pas autant d'importance à la première personne du singulier « moi, je ». Les Slovaques sont de cette culture-là, 
on retrouve là aussi l'influence du régime précédent. Travailler en équipe ne leur pose pas de problème et ils sont beaucoup moins individualistes.

Les Français sont plus sensibles à l'espace personnel de chacun et attendent, en retour, la même distance. Les Slovaques en tant que Slaves appartiennent aux cultures émotives (Gromová - Muglova, 2005: 44). Il est tout à fait normal pour eux de toucher le bras de leur partenaire au cours d'une discussion. Ils manifestent ainsi leur sympathie ou leur accord, ce qui est incompréhensible et inhabituel pour les Français.

En France, le contact physique se manifeste en revanche au moment de l'embrassade en se saluant et en se disant «bonjour, bonsoir ». Le nombre de bises des amis ou des collègues qui se connaissent diffère selon les régions. Cette habitude surprend tous les étrangers y compris les Slovaques. Pour les Français, il est courant de faire la bise à une personne que l'on rencontre pour la première fois, mais cela n'implique pas forcément une marque d'amitié. Il vaut mieux le savoir par avance pour bien le comprendre et bien se comporter.

Nous pouvons constater que le contact physique existe sous différentes formes dans les deux cultures. Il suffit de connaître cette différence, de la comprendre et de la respecter.

Les Français sont polis, ils vouvoient leurs partenaires et ils les appellent par leurs noms, mais très vite ils passent aux prénoms. Cette façon de s'adresser aux personnes semble inhabituel pour les Slovaques et peut être perçu comme un manque de respect et pourrait éventuellement mener à un sentiment d'infériorité.

Une différence nous semble particulièrement importante tant au niveau professionnel que personnel. Les Français accordent une attention particulière à l'hospitalité et à la gastronomie. Peu importe dans quel pays ils se trouvent, ils apprécient beaucoup le fait d'être accueillis au restaurant ou chez un autochtone. Il est très intéressant pour eux de discuter, de mieux connaître les habitudes des Slovaques (ou d'autres), de partager ces moments de convivialité autour d'un repas commun. Nous ne considérons pas ici ce fait comme moins important pour les autres nations mais nous y voyons une différence entre Slovaques et Français. Les Français bougent et déplacent les tables dans les restaurants pour être tous ensemble à table. Ils aiment bien prolonger ces moments de convivialité et de bonne chère.

Nous avons mentionné quelques traits caractéristiques des différences culturelles entre les deux nations. Ces quelques exemples sont cependant loin d'être exhaustifs. Pour avoir une vision plus synthétique de ces différences culturelles, nous renvoyons le lecteur à l'ouvrage de Schmitt (2015) qui analyse le comportement des Français et des Slovaques pour mettre en évidence les fondements sociologiques, psychologiques, voire philosophiques, de leurs différences culturelles. A titre d'exemple, la place primordiale de la rationalité, l'attitude positive face à la vie chez les Français, l'importance du paraitre, l'attitude de fermeture et de retrait chez des Slovaques. Meskova (2012: 51-55) identifie d'autres traits culturels opposés entre ces deux nations, comme la motivation (les manageurs français motivent plus leurs employés, l'approche des manageurs slovaques est plus directive), le pragmatisme (les manageurs slovaques sont plus pragmatiques, ils se décident assez rapidement tandis que les partenaires français préfèrent se prononcer après réflexion), la notion de temps (les manageurs slovaques aiment respecter l'heure comme leurs voisins de culture germanique tandis que les Français sont assez laxistes en ce qui concerne les délais et les rendez-vous; les Slovaques attendent que le programme de la réunion de travail ou de la négociation soit assez strictement respecté), le manque d'assertivité (les Slovaques sont en général caractérisés par la modestie, considérée par les Français comme déplacée car leurs compétences professionnelles et linguistiques sont très appréciées; un certain manque d'assertivité est souvent évident), la dimension émotionnelle (le management slovaque est plus émotionnel, plus empathique). Selon

XLinguae, Volume 11 Issue 1XL, January 2018, ISSN 1337-8384, eISSN 2453-711X 
Lips et Pfizenmaier (2008), la culture française se range plutôt parmi les cultures polychrones et la culture slovaque parmi les cultures monochrones. Elles ne se trouvent pas du même côté sur le spectre des cultures de Hall (1984). Nous ne traiterons pas ici des différences au quotidien (Meskova, 2007: 47-55) qui sont assez nombreuses, par exemple les salutations, les habitudes alimentaires, le relationnel ou encore le savoir-vivre.

Nous constatons que les Français et les Slovaques sont plutôt tolérants et ouverts aux cultures d'autres nations.

Malgré le régime totalitaire précédent et malgré les effets de la société de consommation depuis 1989, les Slovaques gardent un côté humain, émotionnel, l'héritage de "l'âme slave ". C'est un bagage intéressant pour tous les étrangers y compris ceux venant d'autres pays d'Europe occidentale. N'oublions pas que les uns et les autres sont membres d'une même entité, l'Union européenne. Nous partageons l'avis de Côme (2012: 22) qui affirme que pour qu'un habitant d'un des pays membres de l'Union puisse apprécier à sa juste valeur l'opportunité que constitue la diversité culturelle européenne, il lui faut avoir le niveau nécessaire de compréhension et d'acceptation des différences.

\section{L'interculturel dans l'enseignement et dans la vie professionnelle}

Les résultats de nos observations pourraient être utilisés dans le domaine de l'enseignement. Il est nécessaire d'éveiller la sensibilité aux différences culturelles dès les premières années de la scolarité et de la développer par la suite dans l'enseignement secondaire et universitaire. Birová (2017) souligne que les compétences culturelles et interculturelles des apprenants doivent figurer au centre de l'intérêt des enseignants. Höhn (2013: 52) confirme aussi ce besoin en donnant l'exemple concret d'une université slovaque (Université Matej Bel). Pecníková (2017 : 92) et d'autres auteurs comme Kubeková - Cervantes Bonet (2014:107) mettent aussi l'accent sur la nécessité de développer ces compétences. Les élèves et les étudiants formés de cette façon seront mieux préparés à la vie professionnelle. Leur travail pourrait être plus efficace dans des organismes ou entreprises où l'effectif est composé de nationalités ou de cultures différentes.

Il est de notoriété publique que Hofstede a commencé à s'intéresser aux particularités culturelles des pratiques de management interculturel dès les années 1960 (Hofstede, Bollinger, 1987). Aujourd'hui, comme nous le savons, l'interculturel devient une composante incontournable dans la coopération internationale. Les entreprises multinationales devraient se transformer en entreprises interculturelles quel que soit leur degré d'internationalisation, ce que confirme aussi Poliak (2015: 111-114) en analysant l'influence des différences culturelles sur le management tant au niveau des employés qu'au niveau de la culture d'entreprise.

C'est pourquoi, il est important que tous ceux qui rencontrent les représentants d'autres cultures ou qui travaillent avec eux prennent cette réalité en considération. Les compétences interculturelles relatives à l'organisation sont décisives pour gérer la diversité culturelle interne croissante au fil de l'internationalisation (Prime, Usunier, 2012: 419). Le travail au niveau international exige des compétences interculturelles éprouvées : compétences linguistiques, sensibilité interculturelle, gestion de l'incertitude, leadership international, travail en équipes. Selon Prime - Usunier (2012: 410-411, 417), les acteurs du marketing international, en particulier les négociateurs, les expatriés, les chargés de mission ou les chefs de projets multipays sont particulièrement concernés par le développement des compétences interculturelles.

La synergie culturelle qui nous paraît la plus convenable mène le mieux à la sensibilité culturelle de tous ceux qui entrent dans le jeu international. Tout ce qui nous semble naturel dans notre comportement, dans nos habitudes, peut être ressenti différemment par un représentant d'une autre culture. La coopération interculturelle 
prévoit des équipes dans lesquelles chacun peut apporter ce qu'il a de spécifique, de particulier et de meilleur. Équilbey (2004: 21-22,181) conseille de s'ouvrir aux autres cultures, d'inventorier ce que l'on sait et doit savoir sur les autres, de rechercher les erreurs à éviter pour ne pas sombrer dans les préjugés, les stéréotypes et l'ethnocentrisme. L'interculturalité peut favoriser la créativité et le développement personnel, mais elle peut aussi receler un fort potentiel de dysfonctionnements. Il faut apprendre à atténuer certains traits au profit d'autres.

Afin d'accroître l'harmonie et l'efficacité au sein des équipes de travail et d'éviter les tensions culturelles ou les conflits culturels qui constituent l'un des risques importants auxquels doit faire face un leader d'organismes multinationaux, il est nécessaire de se préparer, de se former en avance.

En Slovaquie, les centres de formation n'offrent aux firmes que $8,7 \%$ de cours orientés vers la communication interculturelle en général dont seulement 3,5\% proposent un entraînement intensif dans le domaine de la communication verbale et non verbale, ils apprennent à respecter les différences culturelles, à éviter le choc culturel (Bencikova, Minarova, 2013: 21). Zelenkova (2010: 9-11; 2005; 2014 : 166172) souligne aussi le besoin de formation et d'entraînement des étudiants futurs cadres dirigeants et des manageurs. Il en va de même pour les enseignants et les formateurs qui devraient également se former à la problématique de la communication interculturelle, à la sensibilité culturelle afin d'éviter les malentendus qui peuvent parfois avoir des conséquences désastreuses dans les relations professionnelles au niveau international.

\section{Conclusion}

Il est nécessaire que les représentants de différentes cultures se comprennent et se respectent lors de leurs rencontres, non seulement au niveau international, mais aussi au niveau national, régional et local. La connaissance de l'autre est impossible sans la connaissance de soi.

Il est évident que les différences entre les pays d'Europe occidentale et les pays de l'ancien bloc de l'Est existent. Elles sont dues à plusieurs facteurs : régimes politiques différents, histoire et culture différentes. Les prendre en considération et faire un effort pour les identifier et les comprendre favorise une meilleure coopération et permet la création d'un climat de confiance afin de mieux discuter, de mieux négocier, de mieux produire, de mieux vendre. Nous sommes entièrement d'accord avec Équilbey (2004: 187) qui affirme que l'héritage culturel influence pour longtemps notre vision du temps, de l'espace et des relations entre les hommes et que nous avons en commun un élément essentiel de richesse : la diversité culturelle. Celleci devrait nous unir et non nous séparer.

Il suffit que les cultures se respectent mutuellement pour que les personnes qui se rencontrent et communiquent ensemble parviennent à une synergie culturelle. Nous sommes persuadée que la connaissance de la culture de l'autre est un réel enrichissement. Tout ce qui est différent, particulier peut être plus intéressant, plus attirant, parfois plus motivant pour aboutir à des résultats satisfaisants. Nul ne sait d'ailleurs qui détient les meilleurs atouts pour l'avenir.

\section{Bibliographic references}

BIROVA, J. 2017. La vérification empirique de mise en place des concepts de l'interculturel dans le contexte FLE. Peter Lang. ISBN 978-3-631-73180-2.

BENCIKOVA, D. - MINAROVA, M. 2013. Cultural intelligence as a learning capability for corporate leadership and management. In: Contemporary challenges towards management III, Katowice: Wydawnictwo Uniwersytetu Śląskiego, pp. 1123. ISBN 978-83-226-2188-9.

XLinguae, Volume 11 Issue 1XL, January 2018, ISSN 1337-8384, eISSN 2453-711X 
COME, T. 2012. Economie et management de la diversite culturelle en Europe. In : Thierry Come, Ludmila Meskova (éds.), Management de la diversite culturelle: quels enjeux pour 1'Europe, Bruylant, Bruxelles, Collection «Identités et Cultures en Europe», vol. 2, pp. 21-43. ISBN 978-2-8027-3560-1.

DRUMMOND-GUITEL, V. 2008. Dejouez les pieces des relations interculturelles et devenez un manager de l'international. Le Mans: Gereso Edition. ISBN 978-2915530-39-1.

EQUILBEY, N. 2004. Le management interculturel. Colombelles: Editions EMS. ISBN 2-84769-017-4.

GOULVESTRE, L. 2012. Les cles du savoir etre interculturel. La Plaine Saint-Denis : Affnor. ISBN 978-2-12-465387-4

GROMOVA, E. - MUGLOVA, D. 2005. Kultura - Interkulturalita - Translacia. Nitra: Univerzita Konstantina Filozofa, Filozoficka fakulta. ISBN 80-8050-946-8.

HALL, Edward Twitchell (1984), Le langage silencieux, Seuil, Paris.

HERMESSE, A. 2005. Les differences interculturelles: Source de tensions dans le monde des affaires. www.udi.hec.ulg.ac.be/cours/.../2005_8.pdf

HOFSTEDE, G. - BOLLINGER, D. 1987. Les differences culturelles dans le management. Paris : Editions d'Organisation.

HOHN, E. 2013. Studiengang "Europäische Kulturstudien" an der Geisteswissenschaftlichen Fakultät der Matej - Bel - Universität in Banska Bystrica. In: Acta Linguistica No 9. Banská Bystrica: Belianum, pp. 51-57. ISBN 978-80-5570593-4.

HOREHAJOVA, M. - MARASOVA, J. 2006. Les modifications de principe de la politique sociale en Slovaquie. In: La dynamique des ressources humaines - facteur potentiel d'integration dans l'Europe élargie. Timişoara : Editura Universităţii de Vest, pp. 348-368. ISBN (10) 973-7608-99-2.

KALIKA, M. 2005. Management europeen et mondialisation. Paris: Dunod. ISBN 210- 049152-0.

KUBEKOVÁ, J. - CERVANTES, M. P. 2014. Adquisicion de los conocimientos culturales en la ensenanza de EFE. In: Anuario de estudios filologicos XXXVII/2014 Universidad de Extremadura, pp. 107 -116. ISSN 0210-817.

LIPS, CH. - PFIZENMAIER, A. 2008. Les technologies de l'information et de la communication pour l'enseignement au service de l'interculturel. In : Chovancova Katarina, Rouet Gilles (éds.), Enseigner l'Europe, Enigma, Nitra, pp. 105-127. ISBN 978-80-89132-58-4.

MEIER, O. 2013. Management interculturel. Paris : Dunod. ISBN 10- 2100587463

MESKOVA, L. 2007. Communication interculturelle France - Slovaquie. Banska Bystrica: Univerzita Mateja Bela EF. ISBN 978-80-8083-508-8.

MESKOVA, L. 2012. Le management interculturel franco-slovaque dans le contexte de l'UE. In: Thierry Come, Ludmila Mesková (éds.), Management de la diversité culturelle: quels enjeux pour l'Europe. Bruxelles : Bruylant, Collection «Identités et Cultures en Europe », vol. 2, 2012, pp. 47-55. ISBN 978-2-8027-3560-1.

PECNIKOVA, J. 2017. Rozvoj klucových kompetencii v interkulturnej komunikácii (teoria a prax). In: Cudzie jazyky v premenach casu. Bratislava: Vydavatelstvo EKONOM, pp.88-92. ISBN 978-80-225-4388-0.

POLIAK, P. 2015. Interkulturne rozdiely v manazmente organizacii. Banska Bystrica : Belianum. ISBN 978-80-557-1029-7.

PRIME, N. - USUNIER, J.-C. 2012. Marketing international. Marches, cultures et organisations. Paris : Pearson. ISBN 978-2-7440-7486-8.

RICO DE SOTELO, C. - SERPEREAU, A. 2011. La recherche en communication internationale et communication interculturelle: contours théoriques et méthodologiques d'un champ mouvant. In: Communication internationale et communication interculturelle, Regards epistemologiques et espaces de pratique. 
Christian Agbobli and Gaby Hsab (eds.), Quebec : Presses de l'Universite du Quebec. ISBN 978-2-7605-3118-5.

ROUET, G. - ROSTEKOVA, M. 2013. Regards croises sur la Slovaquie contemporaine. Banska Bystrica: Universite Matej Bel. ISBN 978-80-557-0493-7

SCHMITT, F. 2015. Les cultures française et slovaque : Analyses comparatives de representations sociales. Louvain-la-Neuve: EME. ISBN 978-2-8066-3251-7.

SLATINSKA, A. - PECNIKOVA, J. 2017. Jazyk - kultúra - identita. Banska Bystrica: Belianum, Vydavatelstvo Univerzity Mateja Bela. ISBN 978-80-557-1284-0. STOICIU, G. 2011. La communication interculturelle comme champ d'études. Histoire, carte et territoire. In: Agbobli Christian, Hsab, Gaby (éds.), Communication internationale et communication interculturelle. Quebec: Presses de l'Universite du Quebec. pp. 45-70. ISBN 9782760531185.

SUCHA, Z. 2013. Europska bankova unia v podmienkach financnej globalizacie. In: Soucasná Europa, CES VSE, Praha , XVIII, 02, pp. 167-185. ISSN 1804-1280.

SICKOVA, Z. 2008. O slove a obraze v krestanskom vytvarnom umeni na Slovensku - dovolene a zakazane. In: Slovo - obraz - zvuk. Duchovny rozmer sucasnej kultury. Nitra: Univerzita Konstantina Filozofa. pp. 171-179. ISBN 80 88778-35-2.

THERY, B. 2002. Manager dans la diversité culturelle. Paris: Editions d'Organisation. ISBN 2-7081-2705-5.

ZELENKOVA, A. 2005. Teacher training for cultural understanding: a new challenge. In: University Language Centres: Broadening Horizons, Expanding Networks, Bratislava - Tubingen: Cercles. pp. 307-313. ISBN 978-3-00-025322-5.

ZELENKOVA, A. 2010. Interkulturne vzdelavanie v cudzich jazykoch na vysokej skole. Metody a ich reflexia. Banska Bystrica: Univerzita Mateja Bela, Ekonomicka fakulta. ISBN 978-80-557-0014-4.

ZELENKOVA, A. 2014. Interkulturna kompetencia v kontexte vysokoskolskeho vzdelavania. Banska Bystrica: Belianum. ISBN 978-80-557-0817-1.

Words: 5430

Characters: $37250(20,69$ standard pages $)$

Doc. PhDr. L'udmila Mešková, PhD.

Department of European cultural studies

Faculty of Letters, University Matej Bel, Banská Bystrica

Tajovského 40, 97401 Banská Bystrica

Slovakia

ludmila.meskova@umb.sk

XLinguae, Volume 11 Issue 1XL, January 2018, ISSN 1337-8384, eISSN 2453-711X 